$W_{0}$ contains the rest energy and the kinetic energy of the emitted (absorbed) particle.

There would appear to be little that is new in this alternative possibility, yet it has one property that is particularly attractive in the search for the stereo. chemical basis of protein specificity. We refer to the fact that in the keto. enol interchange it is the hydrogen atom associated with theside-chain carbon

Table 1 gives numerical values of $\zeta_{1}$ and $\zeta_{2}$ for $W_{0}$ lying between 1.5 and $10 m c^{2}$, and Fig. 1 gives the common logarithm of these functions.

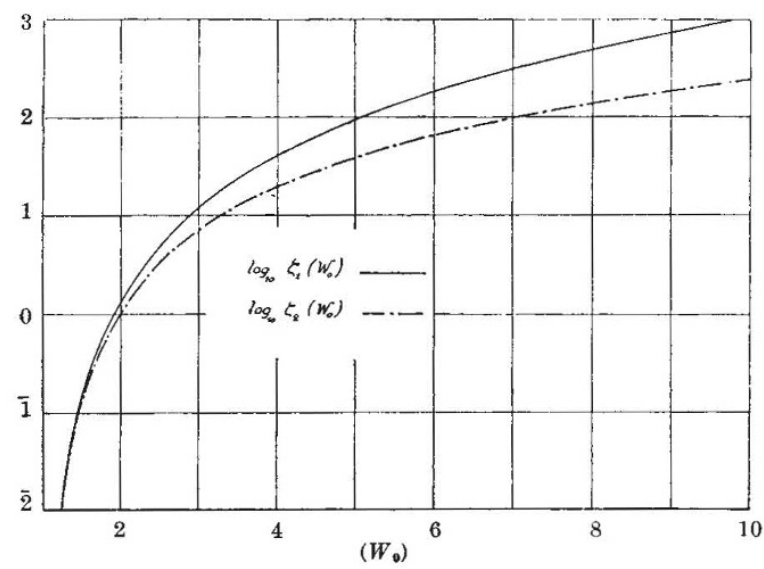

It is of some interest to notice that two experiments would be sufficient to test the above theory, since a single one is enough to determine the constant $C$ :

$$
C=\frac{\lambda \zeta_{1}-10^{-4} Z^{3}}{\lambda \zeta_{2}+10^{-4} Z^{3}},
$$

the experiments being carried out for different values of $W_{0}$ and $Z$, and giving directly the corresponding values of the ratio $\lambda\left(W_{0}, Z\right)$. In the calculation of the ratio $\lambda$, the particular value $C=0$ leads to the hypothesis of Fermi in which $J_{1}$ alone (put equal to unity) enters into the interaction term.

André Mercier.

Universitetets Institut for Teoretisk Fysik,

\title{
Copenhagen.
}

March 31.

1 Fierz, M., Z. Phys., 104, 553 (1937).

${ }^{2}$ See Mercier, A., C.R., 204, 1117, (1937). See also Møller, C., Phys. Rev., 51, 84 (1937).

\section{Intramolecular Folding of Proteins by Keto-Enol Interchange}

Is recent communications ${ }^{1}$ to this and other journals, the hypothesis has been developed of a lactam-lactim interchange $(\mathrm{I})$ to account for the linear folding of polypeptide chains in keratin and myosin and what appears to be a similar, generalized, intramolecular folding in the 'globular' proteins. We wish to point out that in certain respects the argument is unchanged if, instead of a lactam-lactim, a keto-enol interchange (II) is postulated.<smiles>CCC1(C)NCCOC1(C)O</smiles>

I<smiles>CC1NCOCC1(C)O</smiles>

II

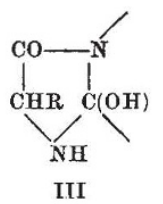

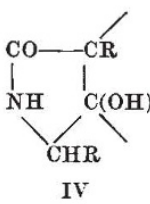

atom that is made use of, and thus we have a possible reason why folds should form only in certain places, as for example in keratin and myosin, the X-ray data and elastic properties of which seem to demand for the $\alpha$-isomer one hexagonal fold for every three amino-acid residues. The responsibility, so to speak, is thrown on to those features of the protein molecule best calculated to bear it, the nature and distribution of the side-chains, and we can imagine certain special sequences acting as a predisposing cause of hydrogen transference at definite intervals and thereby giving rise to specific folds. It may possibly be a drawback of the lactam-lactim interchange that it is perhaps scarcely specific enough.

The general argument is, of course, independent of the exact nature of the hydrogen link involved, whether it be a 'hydrogen bond' 2 or a synchronized oscillation, as recently suggested by Huggins ${ }^{3}$; though actually the latter idea seems to harmonize better with the line of thought traced out here.

There is no need to confine attention to hexagonal folds, either : 5-rings such as III and IV, based also on the lactam-lactim and keto-enol interchange respectively, invite consideration.

Textile Physics Laboratory, University of Leeds.

Mathematical Institute, Oxford.

April 2.

W. T. Astbury.

\section{M. WRINCH.}

${ }^{11}$ Frank, F. C., and Astbury, W. T., J. Text. Inst., 27, P 282 (1936); Chem. Weekbl., 33, 778 (1936). Wrinch, D. M., NATURE, 137, 411 (1936); 138, 241 (1936); Proc. Roy. Soc., $\mathrm{A}$ (in the press). Frank, F. C., NATURE, 138, 242 (1936).

? Jordan Lloyd, D., Biol. Rev., 7, 254 (1932); Jordan Lloyd, D., and Marriott, R. H., Trans. Far. Soc., 29,1228 (1933). Mirsky, A. E., and Pauling, L., Proc. Nat. Acad. Sci., 22, 439 (1936). Wrinch, D. M., and Jordan Lloyd, D., NATURE, 138, 758 (1936).

${ }^{3}$ Huggins, M. L., Nature, 139, 550 (1937).

\section{Anti-Knocks and Pro-Knocks in the Combustion of Fuels}

IT is well known that anti-knocks in concentrations of $0 \cdot 1-0 \cdot 2$ per cent are capable of eliminating detonation completely, whilst they are without influence on the normal combustion regime. This is explicable on the chemical theory of detonation ${ }^{1,2,3}$, according to which detonation can only occur if an intense oxidation reaction has had time to start in the un. burnt mixture. The organic peroxides formed in this stage alter considerably the kinetic parameters and facilitate the production of a detonation wave. Anti-knocks break the reaction chains and hence increase the induction period, thus allowing the mixture to burn before a sufficient concentration of peroxides has been accumulated.

Gusev and Neumann ${ }^{4}$ have shown that two maxima occur in the relation between the average combustion rate and the fuel concentration. The 